\title{
Nonlinear Attitude Estimation Using Active Vision and Inertial Measurements
}

\author{
S. Brás, R. Cunha, J.F. Vasconcelos, C. Silvestre and P. Oliveira
}

\begin{abstract}
In this paper we consider the problem of estimating the attitude of a rigid body equipped with a triad of rate gyros and a pan and tilt camera. The nonlinear attitude observer integrates angular velocity measurements from rate gyros, with images of a planar scene provided by the camera. By exploiting directly sensor information, i) a stabilizing feedback law is introduced and exponential convergence to the origin of the estimation errors is shown; ii) an active vision system is proposed that relies on an image-based exponentially input-tostate stable (ISS) control law for the camera pan and tilt angular rates to keep the features in the image plane. The discrete time implementation of the observer makes use of recent results in geometric numeric integration to preserve the rotation matrix properties. Simulated and experimental results demonstrate the effectiveness and applicability of the proposed solution.
\end{abstract}

\section{INTRODUCTION}

Computer vision has long been recognized as an extremely flexible resource for sensing the environment and acquiring valuable information for pose estimation and control. Visionbased techniques can be seen as a reliable alternative to GPS based navigation for the operation of Unmanned Aerial Vehicles' (UAVs) in indoor and urban environments. The aim of this paper is the development of a nonlinear image based observer to estimate the vehicle attitude relative to a set of image features.

The use of cameras as positioning sensor in control and navigation applications has its most significant representative in the body of work devoted to vision-based control. Over the years, this topic has been extensively studied, experimentally tested, and is well documented (see for example [1] and references therein). The literature on vision-based rigid-body stabilization and estimation highlights important questions and indicates possible solutions to i) keeping feature visibility along the system's trajectories for a large region of attraction [2] ii) minimizing the required knowledge about the 3-D model of the observed object [3], iii) guaranteeing convergence in the presence of camera parametric uncertainty and image measurement noise [3], iv) establishing observability conditions for attitude estimation [4], [5].

In many applications it is desired to design observers based only on the rigid body kinematics, that are an exact description of the physical quantities involved. In this approach, the attitude of the vehicle is propagated by integrating

The authors are with the Institute for Systems and Robotics (ISR), Instituto Superior Técnico, Lisbon, Portugal. E-mails: $\{$ sbras, rita, jfvasconcelos, cjs, pjcro\}aisr.ist.utl.pt Tel: (+351) 21-8418054, Fax: (+351) 21-8418291.

This work was partially supported by Fundação para a Ciência e a Tecnologia (ISR/IST plurianual funding) through the POS Conhecimento Program that includes FEDER funds and by the project PTDC/EEAACR/72853/2006 HELICIM. The work of J.F. Vasconcelos was supported by a PhD Student Scholarship, SFRH/BD/18954/2004, from the Portuguese FCT POCTI programme. inertial sensor measurements [6], [7], [8]. Research on the problem of deriving a stabilizing law for systems evolving on manifolds, where attitude is parameterized, can be found in [9], [10], [11], [12], [13], that provide important guidelines for observer design and discuss the topological limitations to achieving global stabilization on the $\mathrm{SO}(3)$ manifold.

The development of numeric integration methods that preserve geometric properties evolving on Lie groups has witnessed in the last fifteen years a remarkable progress. These methods were originally proposed by Crouch and Grossman in [14]. In [15] the author construct generalized Runge-Kutta methods for integration of differential equations evolving on Lie groups, where the computations are performed in the Lie algebra, which is a linear space. More recently, the work in [16] describes commutator-free Lie group methods to overcome some of the problems associated with the computation of commutators. An application of geometric numeric integration to multi-body dynamics evolving in $\operatorname{SE}(3)$ can be found in [17].

In this work we consider the problem of estimating the attitude of a rigid body equipped with a triad of rate gyros and a pan and tilt camera. By exploiting directly sensor information, a stabilizing feedback law with exponential convergence to the origin of the estimation errors is proposed. As a second goal, we develop an active vision system targeted at keeping the features inside the image plane. For that purpose, an image-based control law for the camera pan and tilt angular rates is proposed. The discrete time implementation of the observer is addressed using recent results from numerical analysis.

The paper is structured as follows. In Section II, the attitude estimation and the camera pan and tilt control problems are introduced. In Section III the attitude observers are presented, and their properties are highlighted. The camera pan and tilt controller is derived in Section IV. A low complexity discrete time implementation of the observer is presented in Section V. In Section VI simulations illustrate the performance of the observer discrete time approximation and the pan and tilt controller. A real time prototype is described in Section VII, and some preliminary experimental results are shown. Concluding remarks and comments on future work are presented in Section VIII.

\section{NOMENCLATURE}

The 3-dimensional special orthogonal group and the special Euclidean group are denoted by $\mathrm{SO}(3)$ and $\mathrm{SE}(3)$, respectively. The notation $\operatorname{diag}(\mathbf{a})$ describes a diagonal matrix formed by placing the elements of $\mathbf{a} \in \mathbb{R}^{n}$ in the main diagonal. A rotation matrix that transforms a vector from frame $\{A\}$ to frame $\{B\}$ is denoted by ${ }_{A}^{B} \mathbf{R}$. The time 

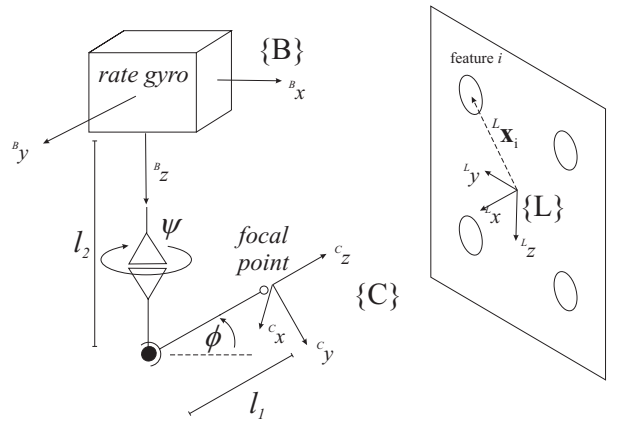

Fig. 1. Diagram of the experimental setup.

dependence of variables will be omitted, unless required for the sake of clarity.

\section{PROBLEM FORMULATION}

Consider a rigid body equipped with a triad of rate gyros and a pan and tilt camera. Let $\{B\}$ be the frame attached to the rigid body, $\{L\}$ the local frame attached to the feature plane, and $\{C\}$ the camera frame with origin at the camera's center of projection with the $z$-axis aligned with the optical axis. The observed scene consists of four points whose coordinates in $\{L\}$ are denoted by ${ }^{L} \mathbf{x}_{i} \in \mathbb{R}^{3}, i \in\{1, \ldots, 4\}$. Without loss of generality, the origin of $\{L\}$ is assumed to coincide with the centroid of the feature points so that $\sum_{i=1}^{4}{ }^{L} \mathbf{x}_{i}=0$.

The image based navigation problem illustrated in Fig. 1 can be summarized as the problem of estimating the attitude of a rigid body given by the rotation matrix from $\{L\}$ to $\{B\}$, denoted as ${ }_{B}^{L} \mathbf{R}$, using images of the feature points and angular velocity readings. An image-based controller for the camera pan and tilt angles will also be considered to keep the features in the image plane.

\section{A. Sensor Suite}

The triad of rate gyros is assumed to be aligned with $\{B\}$ so that it provides measurements of the body angular velocity $\boldsymbol{\omega}_{B}$ corrupted by a constant bias term $\boldsymbol{\omega}_{r}=\boldsymbol{\omega}_{B}+\mathbf{b}_{\omega}$, $\dot{\mathbf{b}}_{\omega}=\mathbf{0}$.

As shown in Fig. 1, the camera can describe pan and tilt motions corresponding to the angles $\psi$ and $\phi$, respectively. As such the rotation matrix from $\{C\}$ to $\{B\}$ is given by

$$
\begin{gathered}
{ }_{C}^{B} \mathbf{R}=\mathbf{R}_{\text {pan }} \mathbf{R}_{\text {tilt }}, \\
\mathbf{R}_{\text {pan }}=\mathbf{R}_{z}(\pi / 2+\psi), \quad \mathbf{R}_{\text {tilt }}=\mathbf{R}_{x}(\pi / 2+\phi)
\end{gathered}
$$

where $\mathbf{R}_{z}(\cdot)$ and $\mathbf{R}_{x}(\cdot)$ denote rotation matrices about the $z$-axis and $x$-axis, respectively. The distances between the tilt rotation axis and the origins of $\{C\}$ and $\{B\}$, are respectively, $l_{1}$ and $l_{2}$.

For simplicity of notation, we denote the configuration of $\{C\}$ with respect to $\{L\}$ by $(\mathcal{R}, \mathbf{p}) \in \mathrm{SE}(3)$, where $\mathcal{R}={ }_{C}^{L} \mathbf{R}$ is the rotation matrix from $\{C\}$ to $\{L\}$ and $\mathbf{p}$ the position of the origin of $\{L\}$ with respect to $\{C\}$. Then, the 3-D coordinates of the features points expressed in $\{C\}$ can be written as $\mathbf{q}_{i}=\mathcal{R}^{T L} \mathbf{x}_{i}+\mathbf{p}, i \in\{1, \ldots, 4\}$ and, using the perspective camera model [5], the 2-D image coordinates of those points $\mathbf{y}_{i} \in \mathbb{R}^{2}$ can be written as

$$
\left[\begin{array}{c}
\mathbf{y}_{i} \\
1
\end{array}\right]=\delta_{i} \mathbf{A q}_{i},
$$

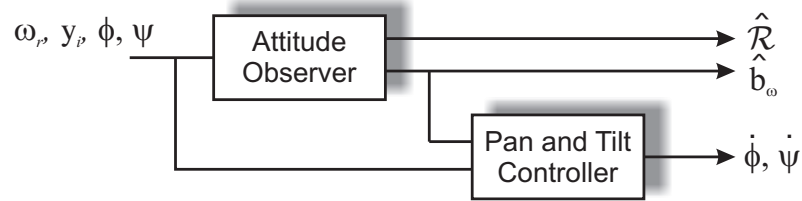

Fig. 2. Block diagram of the attitude observer and camera controller. The quantities $\hat{\mathcal{R}}$ and $\hat{\mathbf{b}}_{\omega}$ are, respectively, the attitude and angular rate bias estimates.

where $\mathbf{A} \in \mathbb{R}^{3 \times 3}$ is the camera calibration matrix assumed to be known and $\delta_{i}$ is an unknown scalar encoding depth information and given by $\delta_{i}=\left(\mathbf{e}_{3}^{T} \mathbf{q}_{i}\right)^{-1}, \mathbf{e}_{3}=\left[\begin{array}{lll}0 & 0 & 1\end{array}\right]^{T}$.

\section{B. Attitude kinematics}

In what follows, we will address the problem of estimating the attitude of the camera frame $\{C\}$ with respect to the local frame $\{L\}$ given by $\mathcal{R} \in \mathrm{SO}(3)$. Assuming that the camera pan and tilt angles are known, we can readily obtain the attitude of the rigid body ${ }_{B}^{L} \mathbf{R}=\mathcal{R}_{B}^{C} \mathbf{R}$ as proposed. The camera frame attitude kinematics can be described by

$$
\dot{\mathcal{R}}=\mathcal{R}[\boldsymbol{\omega}]_{\times},
$$

where once again for simplicity of notation $\omega \in \mathbb{R}^{3}$ denotes the camera angular velocity and $[\mathbf{x}]_{X}$ is the skew symmetric matrix defined by the vector $\mathbf{x} \in \mathbb{R}^{3}$ such that $[\mathbf{x}]_{\times} \mathbf{y}=\mathbf{x} \times \mathbf{y}$, $\mathbf{y} \in \mathbb{R}^{3}$. Taking the time derivative of (1), straightforward computations show that $\boldsymbol{\omega}$ can be written as

$$
\boldsymbol{\omega}={ }_{B}^{C} \mathbf{R} \boldsymbol{\omega}_{B}+\mathbf{R}_{\text {tilt }}^{T}\left[\begin{array}{lll}
\dot{\phi} & 0 & \dot{\psi}
\end{array}\right]^{T},
$$

where $\dot{\psi}$ and $\dot{\phi}$ are the time derivatives of the camera pan and tilt angles, respectively.

\section{Problem Summary}

In summary, the estimation problem addressed in this paper can be stated as follows:

Problem 1: Consider the attitude kinematic model described by (3). Design a dynamic observer for $\mathcal{R}$ based on $\boldsymbol{\omega}_{r}$ and $\mathbf{y}_{i}, i=\{1, \ldots, 4\}$, with the largest possible basin of attraction.

To develop an active vision system using the camera pan and tilt degrees of freedom, we consider the following problem:

Problem 2: Let $\overline{\mathbf{y}}$ be the image of the features' centroid given by $\left[\overline{\mathbf{y}}^{T} 1\right]^{T}=\bar{\delta} \mathbf{A p}, \quad \bar{\delta}=\left(\mathbf{e}_{3}^{T} \mathbf{p}\right)^{-1}$. Design a control law for $\dot{\psi}$ and $\dot{\phi}$ based on $\boldsymbol{\omega}_{r}$ and $\mathbf{y}_{i}, i \in\{1, \ldots, 4\}$, such that $\overline{\mathbf{y}}$ approaches the center of the image plane.

Figure 2 depicts the cascaded composition of the system, where the angular rate bias estimate is fed into the pan and tilt controller.

\section{ATTITUDE OBSERVER}

In the following, we propose a solution to Problem 1 that builds on results presented in [6], where a nonlinear position and attitude observer based on landmark measurements and biased velocity measurements was shown to provide exponential convergence to the origin for the position, attitude, and bias errors. The proposed observer is designed to match the rigid body attitude kinematics taking the form

$$
\dot{\hat{\mathcal{R}}}=\hat{\mathcal{R}}[\hat{\boldsymbol{\omega}}]_{\times},
$$


where $\hat{\mathcal{R}}$ is the estimated camera attitude and $\hat{\omega}$ is the feedback term designed to compensate for the estimation errors.

Some rotational degrees of freedom are unobservable in the case features are all collinear as discussed in [6] and references therein. The following necessary condition for attitude estimation is assumed.

Assumption 1: The features are not all collinear.

We will consider a feedback law for $\hat{\omega}$ that uses measurements of the form

$$
\mathbf{U}=\mathcal{R}^{T}\left[{ }^{L} \mathbf{u}_{1} \ldots{ }^{L} \mathbf{u}_{n}\right] \in \mathbb{R}^{3 \times n},
$$

where ${ }^{L} \mathbf{u}_{i} \in \mathbb{R}^{3}$ are time-invariant in the local frame $\{L\}$. To obtain these vector readings from the image coordinates $\mathbf{y}_{i}$, we explore the geometry of planar scenes. For that purpose, we introduce the matrices

$$
\mathbf{X}=\left[\begin{array}{lll}
{ }^{L} \mathbf{x}_{1} & \ldots & { }^{L} \mathbf{x}_{4}
\end{array}\right], \quad \mathbf{Y}=\left[\begin{array}{ccc}
\mathbf{y}_{1} & \cdots & \mathbf{y}_{4} \\
1 & \cdots & 1
\end{array}\right]
$$

where ${ }^{L} \mathbf{x}_{i}$ are the 3-D coordinates of the feature points expressed in $\{L\}$ and $\mathbf{y}_{i}$ the corresponding 2-D image coordinates. We can now state the following lemma.

Lemma 1: Let $\boldsymbol{\sigma}=\left[\begin{array}{llll}\sigma_{1} & \sigma_{2} & \sigma_{3} & \sigma_{4}\end{array}\right]^{T} \in \mathbb{R}^{4} \backslash\{0\}$ and $\boldsymbol{\rho}=\left[\begin{array}{llll}\rho_{1} & \rho_{2} & \rho_{3} & \rho_{4}\end{array}\right]^{T} \in \mathbb{R}^{4} \backslash\{0\}$ be such that $\mathbf{Y} \boldsymbol{\sigma}=0$, $\mathbf{X} \boldsymbol{\rho}=0$, and $\mathbf{1}^{T} \boldsymbol{\rho}=0$, where $\mathbf{1}=\left[\begin{array}{llll}1 & 1 & 1 & 1\end{array}\right]^{T}$. Consider that the features verify the Assumption 1 and the camera configuration is such that the image is not degenerate (neither a point nor a line). Then, the depth variables $\delta_{i}$ can be written as $\delta_{i}=\alpha \frac{\rho_{i}}{\sigma_{i}}$, where $\alpha \in \mathbb{R}, \rho_{i} \neq 0$, and $\sigma_{i} \neq 0$ for $i \in\{1,2,3,4\}$.

Proof: See [18].

Writing (2) in matrix form and using Lemma 1, we have $\mathbf{Y}=\mathbf{A}\left(\mathcal{R}^{T} \mathbf{X}-\mathbf{p} \mathbf{1}^{T}\right) \alpha \mathbf{D}_{\sigma}^{-1} \mathbf{D}_{\rho}$, where $\mathbf{D}_{\rho}=\operatorname{diag}(\boldsymbol{\rho})$. From the feature centroid constraint $\mathbf{X} 1=0$, it follows that $\alpha \mathcal{R}^{T} \mathbf{X}=\mathbf{A}^{-1} \mathbf{Y} \mathbf{D}_{\rho}^{-1} \mathbf{D}_{\sigma}\left(\mathbf{I}-\frac{1}{4} \mathbf{1 1}{ }^{T}\right)$, which takes the form of (6) up to a scale factor. We can use the properties of the rotation matrix and the positive depth constraint $\delta_{i}>0$ to obtain the normalized vector readings

$$
\overline{\mathbf{x}}_{i}=\mathcal{R}^{T} \frac{{ }^{L} \mathbf{x}_{i}}{\left\|{ }^{L} \mathbf{x}_{i}\right\|}=\operatorname{sign}(\alpha) \frac{\alpha \mathcal{R}^{T L} \mathbf{x}_{i}}{\left\|\alpha \mathcal{R}^{T L} \mathbf{x}_{i}\right\|} .
$$

where $\operatorname{sign}(\alpha)=\operatorname{sign}\left(\frac{\rho_{i}}{\sigma_{i}}\right)$. Finally, we define the matrix $\mathbf{U}$ using linear combinations of (7) so that $\mathbf{U}=\overline{\mathbf{X}} \mathbf{A}_{X}$, where $\mathbf{A}_{X} \in \mathbb{R}^{5 \times 5}$ is nonsingular and $\overline{\mathbf{X}}=\left[\overline{\mathbf{x}}_{1}, \ldots, \overline{\mathbf{x}}_{4}, \overline{\mathbf{x}}_{i} \times \overline{\mathbf{x}}_{j}\right]$ for any linear independent $\overline{\mathbf{x}}_{i}$ and $\overline{\mathbf{x}}_{j}$.

The directionality associated with the features positions is made uniform by defining transformation $\mathbf{A}_{X}$ such that $\mathbf{U U}^{T}=\mathbf{I}$. The desired $\mathbf{A}_{X}$ exists if Assumption 1 is satisfied [6].

Let the bias in angular velocity measurements be constant, i.e. $\dot{\mathbf{b}}_{\omega}=\mathbf{0}$, and consider the Lyapunov function

$$
V=\frac{\|\tilde{\mathcal{R}}-\mathbf{I}\|^{2}}{2}+\frac{1}{2 k_{b_{\omega}}}\left\|\tilde{\mathbf{b}}_{\omega}\right\|^{2}
$$

where $k_{b_{\omega}}>0, \tilde{\mathbf{b}}_{\omega}:=\hat{\mathbf{b}}_{\omega}-\mathbf{b}_{\omega}$, and $\hat{\mathbf{b}}_{\omega}$ is the estimated bias in angular velocity measurements. Its time derivative is given by

$$
\dot{V}=\mathbf{s}_{\omega}^{T}\left(\hat{\boldsymbol{\omega}}-{ }_{B}^{C} \mathbf{R} \boldsymbol{\omega}\right)+\frac{1}{k_{b_{\omega}}} \dot{\tilde{\mathbf{b}}}_{\omega}^{T} \tilde{\mathbf{b}}_{\omega}
$$

where $\mathbf{s}_{\omega}=\mathcal{R}^{T}\left[\tilde{\mathcal{R}}-\tilde{\mathcal{R}}^{T}\right]_{\otimes}$, and $[\cdot]_{\otimes}$ is the unskew operator, such that, $\left[[\mathbf{a}]_{\times}\right]_{\otimes}=\mathbf{a}, \mathbf{a} \in \mathbb{R}^{3}$. The feedback term $\mathbf{s}_{\omega}$ can be expressed as an explicit function of the sensor readings [6, Theorem 8]. Consider the attitude feedback law

$$
\begin{aligned}
& \hat{\boldsymbol{\omega}}={ }_{B}^{C} \mathbf{R}\left(\boldsymbol{\omega}_{r}-\hat{\mathbf{b}}_{\omega}+\mathbf{R}_{\text {pan }}^{T}\left[\begin{array}{lll}
\dot{\phi} & 0 & \dot{\psi}
\end{array}\right]^{T}\right)-k_{\omega} \mathbf{S}_{\omega} \\
& ={ }_{B}^{C} \mathbf{R}\left(\boldsymbol{\omega}-\tilde{\mathbf{b}}_{\omega}\right)-k_{\omega} \mathbf{s}_{\omega},
\end{aligned}
$$

where $k_{\omega}>0$. Applying the feedback law (9) to the Lyapunov function (8) and defining

$$
\dot{\hat{\mathbf{b}}}_{\omega}:=k_{b_{\omega}{ }_{C}} \mathbf{R}_{\mathbf{s}_{\omega}},
$$

the Lyapunov function derivative is given by $\dot{V}=$ $-k_{\omega}\left\|\mathbf{s}_{\omega}\right\|^{2}$.

Considering the feedback law (9) and the differential equation (10), the closed loop attitude error dynamics results in

$$
\begin{aligned}
& \dot{\tilde{\mathcal{R}}}=-k_{\omega} \tilde{\mathcal{R}}\left(\tilde{\mathcal{R}}-\tilde{\mathcal{R}}^{T}\right)-\tilde{\mathcal{R}}\left[\mathcal{R}_{B}^{C} \mathbf{R} \tilde{\mathbf{b}}_{\omega}\right]_{\times} \\
& \dot{\tilde{\mathbf{b}}}_{\omega}=k_{b_{\omega}{ }_{C}^{B}} \mathbf{R} \mathcal{R}^{T}\left[\tilde{\mathcal{R}}-\tilde{\mathcal{R}}^{T}\right]_{\otimes}
\end{aligned}
$$

Lemma 2 provides sufficient conditions for the boundedness of the estimation errors that exclude convergence to the equilibrium points satisfying $\|\tilde{\mathcal{R}}-\mathbf{I}\|^{2}=8$. Global asymptotic stability of the origin is precluded by topological limitations associated with those points [19].

Lemma 2: The estimation errors $\tilde{\mathbf{x}}_{b}=\left(\tilde{\mathcal{R}}, \tilde{\mathbf{b}}_{\omega}\right)$ are bounded. For any initial condition that verifies

$$
\frac{\frac{1}{k_{b_{\omega}}}\left\|\tilde{\mathbf{b}_{\omega}}\left(t_{0}\right)\right\|^{2}}{8-\left\|\tilde{\mathcal{R}}\left(t_{0}\right)-\mathbf{I}\right\|^{2}}<1
$$

the attitude error is bounded and $\|\tilde{\mathcal{R}}(t)-\mathbf{I}\|^{2}<8$ for all $t \geq t_{0}$.

Exploiting the results derived for LTV systems in [20], Theorem 1 establishes the exponential convergence of the system (11) trajectories to the desired equilibrium point.

Theorem 1: Assume that $\boldsymbol{\omega}, \dot{\psi}$ and $\dot{\phi}$ are bounded. Then the attitude error and the bias estimation error converge exponentially fast to the equilibrium point $\left(\tilde{\mathcal{R}}, \tilde{\mathbf{b}}_{\omega}\right)=(\mathbf{I}, 0)$, for any initial condition satisfying (12).

Due to space constraints, the proofs of Lemma 2 and Theorem 1 are omitted. However they can be obtained by adaptation of the derivation used in [6, Lemma 6] and [6, Theorem 7], respectively.

\section{CAmera PAN AND Tilt CONTROLler}

In this section, we address the problem of keeping the features inside the image plane, exploring the camera's ability to describe pan and tilt angular motions. As stated in Problem 2, the strategy adopted to achieve this goal amounts to controlling the camera pan and tilt angular velocities $\dot{\psi}$ and $\dot{\phi}$, using directly the image measurements $\mathbf{y}_{i}$ and the angular velocity readings $\boldsymbol{\omega}_{r}$, so as to keep the image of the features' centroid at a close distance from the center of the image plane.

We resort to Lyapunov theory and consider the following candidate Lyapunov function

$$
W=\frac{1}{2} \mathbf{p}^{T} \Pi \mathbf{p}=\frac{1}{2}\left(p_{x}^{2}+p_{y}^{2}\right),
$$

where $\mathbf{p}=\left[\begin{array}{lll}p_{x} & p_{y} & p_{z}\end{array}\right]^{T}$ is the position of $\{L\}$ expressed in $\{C\}$ and $\Pi \in \mathbb{R}^{3 \times 3}$ is the $x-y$ plane projection matrix. 


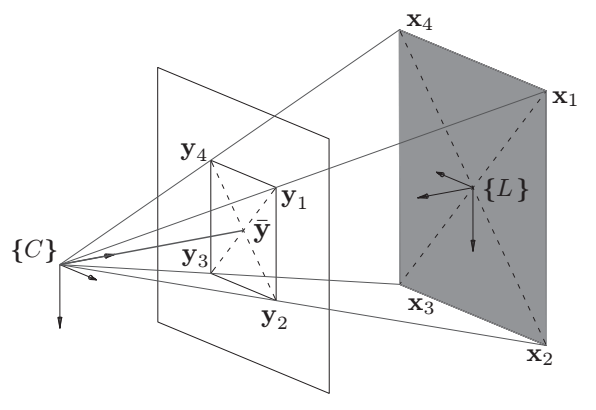

Fig. 3. Projection of the visual features in the image plane.

Using the expression for $\boldsymbol{\omega}$ given in (4), the camera position kinematics can be written as

$$
\begin{aligned}
\dot{\mathbf{p}} & =[\mathbf{p}]_{\times} \boldsymbol{\omega}-\mathbf{v} \\
& =[\mathbf{p}]_{\times}\left(\mathbf{R}_{\text {tilt }}^{T} \mathbf{R}_{\text {pan }}^{T} \boldsymbol{\omega}_{B}+\mathbf{R}_{\text {tilt }}^{T}\left[\begin{array}{lll}
\dot{\phi} & 0 & \dot{\psi}
\end{array}\right]^{T}\right)-\mathbf{v},
\end{aligned}
$$

where $\mathbf{v}$ is the camera linear velocity. Recall that by definition $\mathbf{p}$ coincides with the position of the features' centroid and its image is given by $\overline{\mathbf{y}}$. Therefore, by guaranteeing that the Lyapunov function $W$ is decreasing, or equivalently $\left[\begin{array}{ll}p_{x} & p_{y}\end{array}\right]$ is approaching the origin, we can ensure that $\overline{\mathbf{y}}$ is approaching the center of the image plane. To simplify the notation and without loss of generality, assume from now on that $\mathbf{A}=\mathbf{I}$ so that $\bar{y}_{x}=p_{x} / p_{z}$ and $\bar{y}_{y}=p_{y} / p_{z}$.

Before proceeding to define the pan and tilt control law, we highlight the fact that $\overline{\mathbf{y}}$ can be easily obtained from the image measurements $\mathbf{y}_{i}$. By noting that the feature centroid lies at the intersection between the vectors $\mathrm{x}_{3}-\mathrm{x}_{1}$ and $\mathrm{x}_{4}-$ $\mathbf{x}_{2}$ and the intersection between lines is clearly an image invariant, we can immediately conclude that $\overline{\mathbf{y}}$ coincides with the point at the intersection between $\mathbf{y}_{3}-\mathbf{y}_{1}$ and $\mathbf{y}_{4}-\mathbf{y}_{2}$ (see Fig. 3).

Lemma 3: Let the camera position kinematics be described by (14) and assume that the rigid body and camera motions are such that $p_{z}>0$ and $\cos \phi \neq 0$. Consider the control law for the camera pan and tilt angular velocities given by

$$
\left[\begin{array}{c}
\dot{\phi} \\
\dot{\psi}
\end{array}\right]=k_{c}\left[\begin{array}{cc}
0 & -1 \\
\frac{1}{\cos \phi} & 0
\end{array}\right] \overline{\mathbf{y}}-\left[\begin{array}{ccc}
1 & 0 & 0 \\
0 & \tan \phi & 1
\end{array}\right] \mathbf{R}_{\mathrm{pan}}^{T} \hat{\boldsymbol{\omega}}_{B},
$$

where $\hat{\boldsymbol{\omega}}_{B}=\boldsymbol{\omega}_{r}-\hat{\mathbf{b}}_{\omega}$ and $k_{c}>0$. Then, the time derivative of the Lyapunov function $W$ along the system trajectories satisfies

$$
\dot{W} \leq-\left(k_{c}-\epsilon\right) W, \quad \forall\|\Pi \mathbf{p}\| \geq \frac{1}{\epsilon}\left(\|\Pi \mathbf{v}\|+p_{z}\left\|\tilde{\mathbf{b}}_{w}\right\|\right),
$$

and $0<\epsilon<k_{c}$.

Proof: Taking the time derivative of (13) and using the expressions for $\dot{\mathbf{p}}$ given in (14), we obtain

$$
\begin{aligned}
\dot{W} & =\mathbf{p}^{T} \Pi\left(p_{z}\left[\mathbf{e}_{3}\right]_{\times} \boldsymbol{\omega}-\mathbf{v}\right) \\
& =p_{z}\left[\begin{array}{lll}
p_{y}-p_{x} & 0
\end{array}\right] \mathbf{R}_{\text {tilt }}^{T}\left(\mathbf{R}_{\text {pan }}^{T} \boldsymbol{\omega}_{B}+\left[\begin{array}{lll}
\dot{\phi} & 0 & \dot{\psi}
\end{array}\right]^{T}\right)-\mathbf{p}^{T} \Pi \mathbf{v} .
\end{aligned}
$$

Choosing $\dot{\phi}$ and $\dot{\psi}$ such that

$$
\mathbf{R}_{\text {tilt }}^{T}\left(\mathbf{R}_{\text {pan }}^{T} \hat{\boldsymbol{\omega}}_{B}+\left[\begin{array}{lll}
\dot{\phi} & 0 & \dot{\psi}
\end{array}\right]^{T}\right)=-k_{c}\left[\begin{array}{lll}
-\bar{y}_{y} & \bar{y}_{x} \kappa
\end{array}\right]^{T},
$$

for some $\kappa$ and noting that $\boldsymbol{\omega}_{B_{\tilde{\mathbf{b}}}}=\hat{\boldsymbol{\omega}}_{B}-\tilde{\mathbf{b}}_{w}$ yields $\dot{W}=$ $-k_{c} W-\mathbf{p}^{T} \Pi\left(\mathbf{v}+p_{z}\left[\mathbf{e}_{3}\right]_{\times}{ }_{B}^{C} \mathbf{R} \tilde{\mathbf{b}}_{w}\right)$ and consequently (16) holds. Solving (17) for $\dot{\phi}, \dot{\psi}$, and $\kappa$, we obtain the control law (15).

Remark 1: If we apply the control law (15) to the system with state $\Pi \mathbf{p}=\left[\begin{array}{ll}p_{x} & p_{y}\end{array}\right]^{T}$ and interpret $\mathbf{v}$ and $p_{z} \tilde{\mathbf{b}}_{w}$ as inputs, it follows from (16) that the system is exponentially input-tostate stable (ISS). As such, the distance between the image of the centroid $\overline{\mathbf{y}}$ and the origin is ultimately bounded by $\left\|\Pi \mathbf{v} / p_{z}\right\|$ and $\left\|\mathbf{b}_{w}\right\|$ and converges exponentially fast to that bound. Moreover, if $\Pi \mathbf{v} / p_{z}$ and $\tilde{\mathbf{b}}_{w}$ converge to zero so does $\overline{\mathbf{y}}$.

\section{DISCRETE TIME IMPLEMENTATION}

In this section we describe a procedure to implement the attitude observer proposed in Section III in discrete time.

Classic Runge-Kutta methods cannot be correctly applied to rotation matrix dynamics since they are not able to preserve polynomial invariants like the determinant [21, Theorem IV.3.3]. An alternative is to apply a method that preserves orthogonality, like a Lie group integrator.

The attitude observer dynamics is composed by differential equations (5) and (10), evolving in $\mathrm{SO}(3)$ and $\mathbb{R}^{3}$, respectively. The first is integrated resorting to geometric numeric integration methods namely, the Crouch-Grossman Method (CG) [14], the Munthe-Kaas Method (MK) [15], and the Commutator-Free Lie group Method (CF) [16]. The second is implemented in discrete time using a classical numeric integration technique.

The presented geometric numerical integration algorithms require the knowledge of the function $\hat{\boldsymbol{\omega}}(t)$ at instants between sampling times. Different sampling and computation strategies can be adopted to obtain an approximation of this function using methods such as polynomial interpolation of the sampled data. In the present work, the unit is equipped with tactical grade inertial sensors and computational resources are limited, then $\hat{\boldsymbol{\omega}}$ is linearly interpolated in the interval $[(k-1) T, k T]$, where $T$ is the sample period.

Due to the adopted interpolation, the use of integration methods with order higher than two does not improve the methods accuracy, hence we narrow our analysis to second order methods. The complexity required to implement each step of the second order CG and MK methods, is summarized in Table I, for the operations in $\mathrm{SO}(3)$, exponential map (Exp), inverse of the differential of the exponential map $\left(\right.$ Dexp $\left.^{-1}\right)$, and $3 \times 3$ matrix multiplication (mmult), as defined in [17]. The coefficients for these methods can be obtained in [21] and [17]. Note that there is no second order CF method and higher orders imply higher computational cost, hence it was not included in Table I. Due to its lower computational cost, the second order CG method is selected.

TABLE I

COMPLEXITY IN EACH STEP FOR CG, MK AND LC METHODS.

\begin{tabular}{|c|ccc|}
\hline operation & Exp & $\operatorname{Dexp}^{-1}$ & mmult \\
\hline$C G 2^{\text {nd }}$ order & 1 & 0 & 1 \\
$M K 2^{\text {nd }}$ order & 1 & 1 & 2 \\
\hline
\end{tabular}

The discrete time implementation of equation (10) was obtained by using a second order Adams-Moulton Method, see [22] for further details. This selection was done based on 

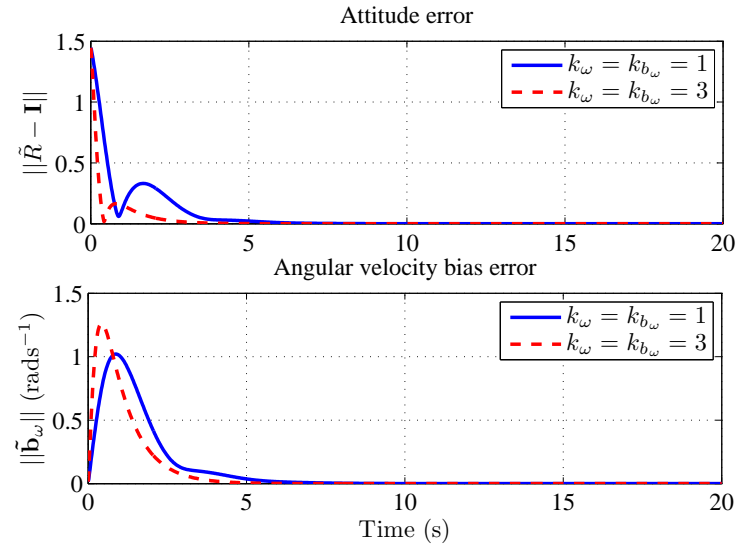

Fig. 4. Attitude observer estimates for two different set of gains.

arguments similar those used for (5). The resulting numerical integration algorithm can be summarized as

$$
\begin{gathered}
\hat{\mathbf{b}}_{\omega k}=\hat{\mathbf{b}}_{\omega k-1}+\frac{T}{2}\left(k_{b_{\omega}{ }_{C}^{B}} \mathbf{R}_{k} \mathbf{s}_{\omega k}+k_{b_{\omega}{ }_{C}^{B}} \mathbf{R}_{k-1} \mathbf{S}_{\omega k-1}\right), \\
\mathcal{R}_{k}=\mathcal{R}_{k-1} \operatorname{Exp}\left(T K^{(1)}\right), \quad K^{(1)}=[\hat{\boldsymbol{\omega}}(k T-T / 2)]_{\times}, \\
\text {where } \hat{\boldsymbol{\omega}}(k T-T / 2) \approx \frac{1}{2}(\hat{\boldsymbol{\omega}}(k T-T)+\hat{\boldsymbol{\omega}}(k T)) . \\
\text { VI. SIMULATION }
\end{gathered}
$$

To assess the effectiveness of the proposed ensemble, this section illustrates, in simulation, the dynamic behavior of the active camera pan and tilt controller and the discrete time implementation of the attitude observer about a typical vehicle maneuver. The tuning capabilities of the observer and the controller are also displayed for two sets of feedback gains.

In the simulation, the positions of the features are ${ }^{L} \mathbf{x}_{1}=$ $\left[\begin{array}{lll}0 & -1 & -1\end{array}\right]^{T} \mathrm{~m},{ }^{L} \mathbf{x}_{2}=\left[\begin{array}{lll}0 & 1 & -1\end{array}\right]^{T} \mathrm{~m},{ }^{L} \mathbf{x}_{3}=\left[\begin{array}{lll}0 & -1 & 1\end{array}\right]^{T} \mathrm{~m}$, and ${ }^{L} \mathbf{x}_{4}=\left[\begin{array}{lll}0 & 1 & 1\end{array}\right]^{T} \mathrm{~m}$, that satisfy $\sum_{i=1}^{4}{ }^{L} \mathbf{x}_{i}=0$ and Assumption 1. The distances $l_{1}$ and $l_{2}$ are set to $0.1 \mathrm{~m}$, and $0.2 \mathrm{~m}$, respectively. The vehicle simulated trajectory is characterized by a circular motion parallel to the ${ }^{L} x,{ }^{L} y$, plane with a radius of $2 \mathrm{~m}$. The associated centrifugal acceleration is aligned with the direction of the ${ }^{B} z$ axis, the initial velocity is $-4 \pi / 12.5 \mathrm{~m} \mathrm{~s}^{-1}$ along the ${ }^{B} y$ axis, and at time $10 \mathrm{~s}$ the velocity decreases linearly during $5 \mathrm{~s}$, reaching zero when the vehicle reaches the starting point of the maneuver. The observer sample time is set to $0.02 \mathrm{~s}$.

The initial estimation errors in the simulations are $\|\mathcal{R}(0)-\mathbf{I}\|=1.4460, \tilde{\mathbf{b}}_{\omega}(0)=\frac{\pi}{180}\left[\begin{array}{lll}0.5 & 0.5 & 0.5\end{array}\right]^{T} \mathrm{rad}$, and the initial pan and tilt camera angles are both set to $20 \frac{\pi}{180} \mathrm{rad}$, thus, (12) is satisfied by the initial conditions.

Figure 4 illustrates the stability and the convergence of the estimation errors of the observer discrete time implementation, validating the results of the Section III. The time evolution of the norm of the center of the features, $\|\overline{\mathbf{y}}\|$, and the actuation imposed by the camera pan and tilt controller are shown in Fig. 5. The overshoot on $\|\overline{\mathbf{y}}\|$ is due to the initial bias estimation error. Notice that, as expected, when the camera linear velocity is non zero the center of the features in the image plane differs from the center of the image. The figures also show that the feedback gains can be
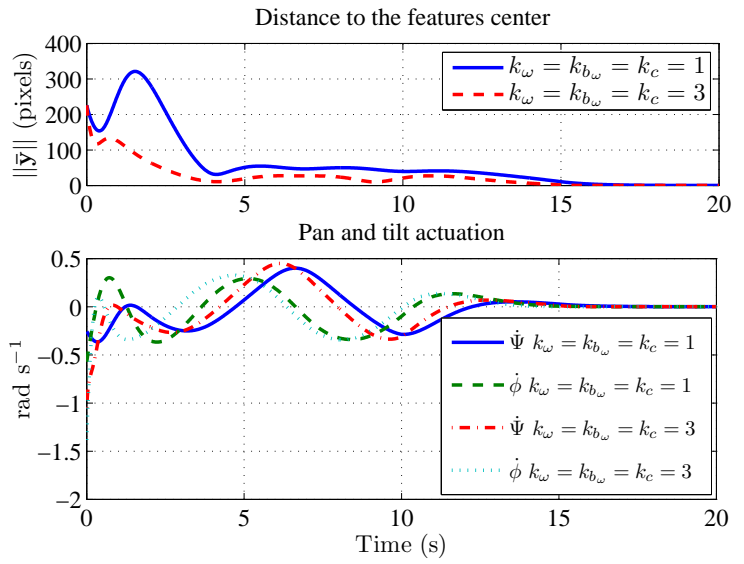

Fig. 5. Distance between the features center and the image center and the pan and tilt actuation for two different set of gains.

used to tune the convergence characteristics of the observer and the controller.

\section{EXPERIMENTAL RESULTS}

In this section we describe the practical implementation of the proposed observer and camera pan and tilt controller. The experimental setup used is composed by a MemSense nIMU, that provides the angular velocity measurements and an AXIS 215 PTZ Camera. The sensor measurements are provided to the estimator without any pre-filtering. The features considered consist of circles segmented by color and placed in ${ }^{L} \mathbf{x}_{1}=\left[\begin{array}{lll}0 & -0.5 & -0.3\end{array}\right]^{T} \mathrm{~m},{ }^{L} \mathbf{x}_{2}=\left[\begin{array}{lll}0 & 0.5 & -0.3\end{array}{ }^{T} \mathrm{~m}\right.$, ${ }^{L} \mathbf{x}_{3}=\left[\begin{array}{llll}0 & -0.5 & 0.3\end{array}\right]^{T} \mathrm{~m}$ and ${ }^{L} \mathbf{x}_{4}=\left[\begin{array}{lll}0 & 0.5 & 0.3\end{array}\right]^{T} \mathrm{~m}$. The distances $l_{1}$ and $l_{2}$ of the AXIS 215 PTZ are $0 \mathrm{~m}$, and $0.088 \mathrm{~m}$, respectively. The system sampling frequency is set to $10 \mathrm{~Hz}$ due the time constrains on the communication with the camera and the image processing time. In the following, and for the sake of readability, the estimator results are displayed using Z-Y-X Euler angles, roll, pitch, and yaw, from frame $\{B\}$ to frame $\{L\}$.

The selected gains are $k_{\omega}=1, k_{b_{\omega}}=0.01$, and $k_{c}=0.5$. Notice that the use of high values for the gains $k_{b_{\omega}}$ gives better bias estimation characteristics but reduces the accuracy of the attitude estimates, since the estimator tends to amplify the measurements noise. Therefore, a compromise needs to be considered, function of the sensors noise characteristics and the desired estimator performance.

The experimental setup only guarantees a reliable ground truth to the ${ }^{B} z$ axis, hence the experiment consisted in rotating the system about this axis. The rotation takes place at time $t=84 \mathrm{~s}$. The initial roll, pitch, and yaw angles are $0 \mathrm{rad}, 0 \mathrm{rad}$, and $-2 \pi / 180 \mathrm{rad}$, respectively, and the final are $0 \mathrm{rad}, 0 \mathrm{rad}$, and $-45 \pi / 180 \mathrm{rad}(0.7854 \mathrm{rad})$, respectively. The initial camera pan and tilt angles are set to zero.

The time evolution of the attitude and bias estimation is shown in the Fig. 6. It is clear the attitude estimate converges to the real attitude when the system is disturbed and the rate gyros bias reaches a steady state. Furthermore, we highlight the overall accuracy of the estimates. The standard deviation of the roll, pitch and yaw angles in the first $84 \mathrm{~s}$ of 

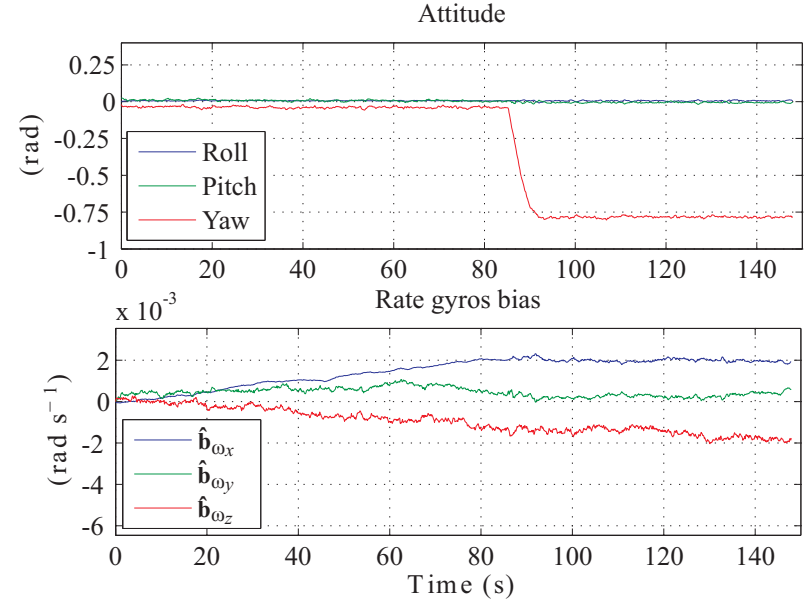

Fig. 6. Experimental data obtain by the observer prototype.
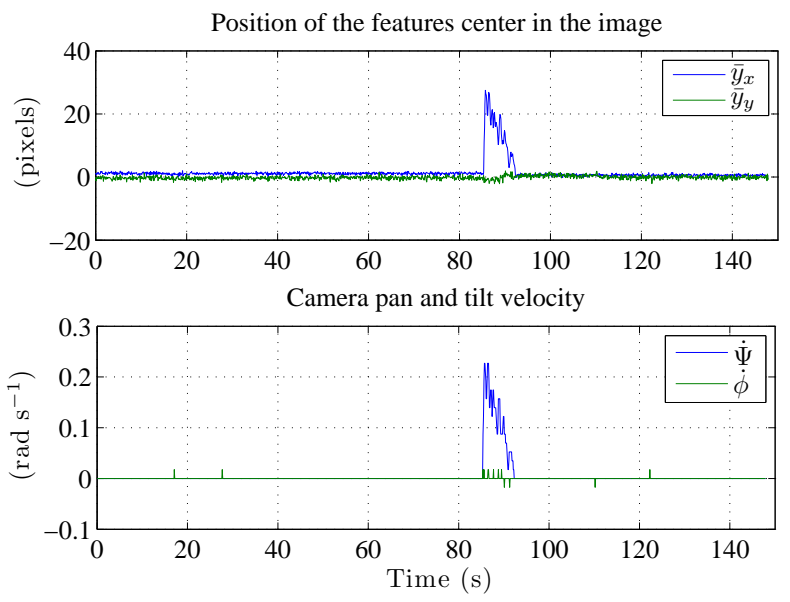

Fig. 7. Time evolution of the position of the center of the features in the image coordinates and the camera pan and tilt velocities.

the experiment are $0.0842 \pi / 180 \mathrm{rad}, 0.2989 \pi / 180 \mathrm{rad}$, and $0.3466 \pi / 180 \mathrm{rad}$, respectively.

Figure 7 depicts the position of the center of the features relatively to the image center, and the camera pan and tilt velocity. As predicted by the theoretical results the actuation increases with the error, and the error converges to zero when the system is in a stationary position. Due to the fact that camera pan and tilt velocity commands are integer values of degrees per second the actuation signals are quantized.

\section{CONCLUSIONS}

This paper addressed the problem of estimating the attitude of a rigid body equipped with a triad of rate gyros and a pan and tilt camera. Based only on the position of four features in the image plane and biased angular velocity measurements, the error estimates obtained by the observer converge exponentially fast to the origin. In order to keep the features in image, an exponentially ISS nonlinear controller for the pan and tilt camera angles was proposed. Simulations were presented to illustrate the dynamic behavior of the overall solution. A simple experimental evaluation with a real time prototype exhibited good performance and attested the applicability of the proposed technique.

\section{ACKNOWLEDGMENTS}

The authors gratefully acknowledge Prof. Brynjulf Owren from the Norwegian University of Science and Technology for sharing his insight on geometric integration algorithms.

\section{REFERENCES}

[1] O. Tahri and F. Chaumette, "Point-based and region-based image moments for visual servoing of planar objects," IEEE Transactions On Robotics, vol. 21, no. 6, pp. 1116-1127, 2005.

[2] N. Cowan, J. Weingarten, and D. Koditschek, "Visual servoing via navigation functions," IEEE Transactions on Robotics and Automation, vol. 18 , no. 4, pp. 521-533, 2002.

[3] E. Malis and F. Chaumette, "Theoretical improvements in the stability analysis of a new class of model-free visual servoing methods," IEEE Transactions on Robotics and Automation, vol. 18, no. 2, pp. 176-186, 2002.

[4] A. P. Aguiar and J. Hespanha, "Minimum-energy state estimation for systems with perspective outputs," IEEE Transactions on Automatic Control, vol. 51, no. 2, pp. 226-241, 2006.

[5] H. Rehbinder and B. K. Ghosh, "Pose estimation using line-based dynamic vision and inertial sensors," IEEE Transactions on Automatic Control, vol. 48, no. 2, pp. 186-199, 2003.

[6] J. Vasconcelos, R. Cunha, C. Silvestre, and P. Oliveira, "Landmark based nonlinear observer for rigid body attitude and position estimation," in 17th IFAC World Congress, South Korea, Seoul, Jul. 2008.

[7] R. Mahony, T. Hamel, and J. M. Pflimlin, "Nonlinear complementary filters on the special orthogonal group," IEEE Transactions on Automatic Control, vol. 53, no. 5, pp. 1203-1218, Jun. 2008.

[8] J. Thienel and R. M. Sanner, "A coupled nonlinear spacecraft attitude controller and observer with an unknown constant gyro bias and gyro noise," IEEE Transactions on Automatic Control, vol. 48, no. 11, pp. 2011-2015, Nov. 2003.

[9] N. Chaturvedi and N. McClamroch, "Almost global attitude stabilization of an orbiting satellite including gravity gradient and control saturation effects," in 2006 American Control Conference, Minnesota, USA, Jun. 2006

[10] D. Fragopoulos and M. Innocenti, "Stability considerations in quaternion attitude control using discontinuous Lyapunov functions," IEE Proceedings on Control Theory and Applications, vol. 151, no. 3, pp. 253- 258, May 2004.

[11] D. E. Koditschek, "The Application of Total Energy as a Lyapunov Function for Mechanical Control Systems," Control Theory and Multibody Systems, vol. 97, pp. 131-151, 1989.

[12] M. Malisoff, M. Krichman, and E. Sontag, "Global stabilization for systems evolving on manifolds," Journal of Dynamical and Control Systems, vol. 12, no. 2, pp. 161-184, Apr. 2006.

[13] S. Salcudean, "A globally convergent angular velocity observer for rigid body motion," IEEE Transactions on Automatic Control, vol. 36, no. 12, pp. 1493-1497, Dec. 1991.

[14] P. E. Crouch and R. Grossman, "Numerical integration of ordinary differential equations on manifolds," J. Nonlinear Science, vol. 3, pp. $1-33,1993$

[15] H. Z. Munthe-Kaas, "Runge-Kutta methods on Lie groups," BIT, vol. 38 , no. 1, pp. 92-11, 1998.

[16] E. Celledoni, A. Marthinsen, and B. Owren, "Commutator-free Lie group methods," Future Generation Computer Systems, vol. 19, no. 3, pp. 341-352, Apr. 2003.

[17] J. Park and W.-K. Chung, "Geometric integration on Euclidean group with application to articulated multibody systems," IEEE Transactions on Robotics, vol. 21, no. 5, pp. 850-863, Oct. 2005.

[18] R. Cunha, "Advanced motion control for autonomous air vehicles," Ph.D. dissertation, Instituto Superior Técnico, Lisbon, 2007.

[19] S. P. Bhat and D. S. Bernstein, "A topological obstruction to continuous global stabilization of rotational motion and the unwinding phenomenon," Systems and Control Letters, vol. 39, no. 1, pp. 63-70, Jan. 2000.

[20] A. Loría and E. Panteley, "Uniform exponential stability of linear timevarying systems: revisited," Systems and Control Letters, vol. 47, no. 1, pp. 13-24, Sep. 2002.

[21] E. Hairer, C. Lubich, and G. Wanner, Geometric Numerical Integration, Structure-Preserving Algorithms for Ordinary Differential Equations, 2nd ed., ser. Springer Series in Computational Mathematics. Springer, 2006, vol. 31.

[22] R. Burden and J. Faires, Numerical Analysis. Boston: PWS-KENT Publishing Company, 1993. 